\title{
An Adaptive and Interactive Agent Based ITS for Cognitive Skill Prediction and Improvement
}

\author{
${ }^{1}$ Mrs.R.Gowri, ${ }^{2}$ Dr.S.Kanmani, ${ }^{3}$ M.Santhosh, ${ }^{4}$ S.Naresh \\ 1 Associate Professor, Department of IT, Sri Manakula Vinayagar Engineering College, Pondicherry. \\ gowrithirugnanam@gmail.com \\ ${ }^{2}$ Professor, Department of IT, Pondicherry Engineering College, Pondicherry. \\ kanmani@pec.edu \\ ${ }^{3,4}$ Students, Department of IT, Sri Manakula Vinayagar Engineering College, Pondicherry. \\ santhoshm.it.2010@gmail.com \\ naresh.smvec@gmail.com
}

\section{ABSTRACT}

This paper proposes an adaptive and interactive agent based intelligent tutoring system for cognitive ability realization and improvement (ITSCARE - Intelligent Tutoring System for Cognitive Ability Realization and Improvement). ITSCARE allows the learners to realize their cognitive ability and to improve their cognition while studying the course. It provides different types of course materials which are dynamically adapted. It also increases the confidence level of the learners and provides an effective learning experience. This system also provides game based learning which influences the learners to get motivated and focus on the course. After the completion of each chapter, a test is conducted to predict the cognitive ability where students are assessed using their help seeking skills during the test and cognitive skill factors such as memory, concentration, attention in detail etc,. It uses politeness style to provide the test results and feed back to the students which keep the learner interest in the subject. Collaborative learning among the learner is improved by conducting quiz competition where a group of students participate and a winner is chosen. It uses different type of software agent to predict and improve the cognitive skill.

Index terms - Intelligent Tutoring System, Interactive Learning Environment, Teaching and Learning Strategies, Cognitive Tutors, Agent based Intelligent Tutoring System, Competition based ITS, Education, Cognitive Prediction and Improvement, Game based ITS.

\section{INTRODUCTION}

An intelligent tutoring system (ITS) [2][5] provides personalized instruction or feedback to students, that is, without the intercession of human tutor. Instead of acquiring a tutor to learn a skill, the user will acquire a software application with "lightweight tutoring agents" that can be engaged to support learning in the environment.

An extension of ITS is Intelligent Tutoring System for Cognitive ability Realization and Improvement (ITSCARE) that we proposed which provides learning environment to realize and improves the cognitive ability of the student and also helps to improve subjective knowledge. The cognitive skill of the student can be predicted based on their help seeking skill and many parameters like memory, understanding \& planning and problem solving during the test.

In this paper, Section 2 introduces background work of intelligent tutoring systems, and the Index of Learning Styles, Section 3 gives the prior research. Section 4 introduces the ITSCARE, and Section 5 outlines the conclusions and future work

\section{BACKGROUND WORKS}

\subsection{Intelligent Tutoring System}

Intelligent Tutoring Systems (ITS) [2] [5] are adaptive systems which use many technologies to personalize learning of student according to the individual student characteristics, such as learning style, mood and knowledge of the subject. There are three main approaches to intelligent tutoring [2]:

- Curriculum sequencing that introduces adaption by presenting learners with material in a sequence and styles that suited to their need.

- Intelligent solution analysis that adds intelligence to intelligent tutoring system by giving students comprehensive feedback on incomplete or mistaken solutions, helps to learn from their blunder.

- Problem solving support techniques that gives learners intelligent assistance to reach a solution

Generally ITS comprises of four tutoring process modules, each will perform different task in tutoring environment.

\subsubsection{Student module}

This module should model the individual characteristics of the student, among then one of the most important is the instant individual knowledge about the domain, Robles defines it as: "The model of the student reflects how much does the student know about the domain, as well as its cognitive experiences and of learning, from which a diagnostic can be made". Besides it should be considered that this module will have to be able to interact with its pair, which a fundamental characteristic of intelligent advisors.

\subsubsection{Tutor's module}


This module posses the knowledge about teaching strategies and tactics, in order to choose then regarding the characteristics of the student (which are stored in the student module).

\subsubsection{Domain's module}

This module posse the knowledge of the subject formed by the production rules, stereotypes, etc. From this module the tutor can obtain the knowledge to be imparted.

\subsubsection{Interface module}

This is the interface between the ITS and the real student, its function is to present the domain's material and any other didactical material in a proper way.

\subsection{Index of Learning Style (ILS)}

The Index of Learning Styles (ILS) model [2] [7] explains the teaching and learning styles in engineering education. It represents an individual student's learning style as in form of four dimensions that specifies both the nature of their learning style preference and the strength. Each learning style dimension relates to a step in the process of receiving and processing of information, as illustrated in Fig.1 In addition to the formal assessment questionnaire, the ILS model describes typical learner behaviors that can be used to informally group types of learners. The ILS model was adopted in the implementation of ITSCARE for predicting the learning style of the student to provide content adaptation.

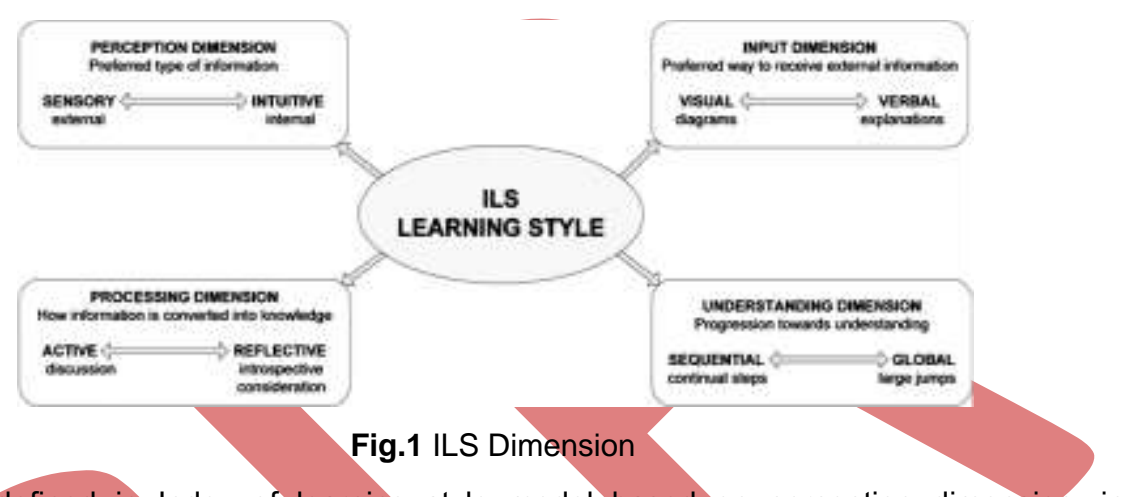

The learner's behavior defined in Index of learning style model based on perception dimension, input dimension, processing dimension and understanding dimension are given in table

Table.1 Typical learner behavior characteristics extracted from the ILS model

\begin{tabular}{l|l}
\multicolumn{1}{c|}{ Sensor } & \multicolumn{1}{c}{ Intuitor } \\
Prefer facts, data, experimentation & Prefer principles and theories \\
Prefer solving problems using standard methods & Prefer innovation \\
Dislike surprises & Dislike repetition \\
Patient with detail & Bored by detail \\
Do not like complications & Welcome complications \\
Good at memorising facts & Good at grasping new concepts \\
Careful but slow & Quick but careless \\
Comfortable with symbols (eg. words) & Uncomfortable with symbols \\
\hline Visual &
\end{tabular}

Remember what they see

Like pictures, diagrams, flow charts, time lines, films

Prefer visual demonstration

Remember what they hear, or what they hear then say Like discussion

Prefer verbal explanation

Learn by explaining to others

\section{Active}

Do something with information - discuss/explain/test Active experimentation

Do not learn much in passive situations (lectures)

Work well in groups

Experimentalists

Process information by setting up an experiment to test an idea,

or try out on a colleague

\section{Sequential}

Follow linear reasoning processes

Can work with material they have only partially or superficially

understood

Strong in convergent thinking and analysis

Learn best when information is presented in a steady progression

of complexity and difficulty 


\section{PRIOR RESEARCH}

\subsection{Conversational ITS to automatically predict learning styles}

Oscar is a novel conservation intelligent tutoring system (CITS) [2] that leads a tutoring conversation and dynamically predicts and adapts to a student's learning style. A CITS that can imitate a human tutor by a better leading an adaptive tutorial conservation uses a familiar format which can help improve learner confidence and motivation. Imitation of human is done by using knowledge of learning style and learning behavior to predict the learning style.

Here a series of experiment is done that determine that prediction of learning style from the learner's behavior during a tutorial conversation and to propose a methodology and architecture. An Example logic rules to adjust student learning style values based on tutoring conversation is given

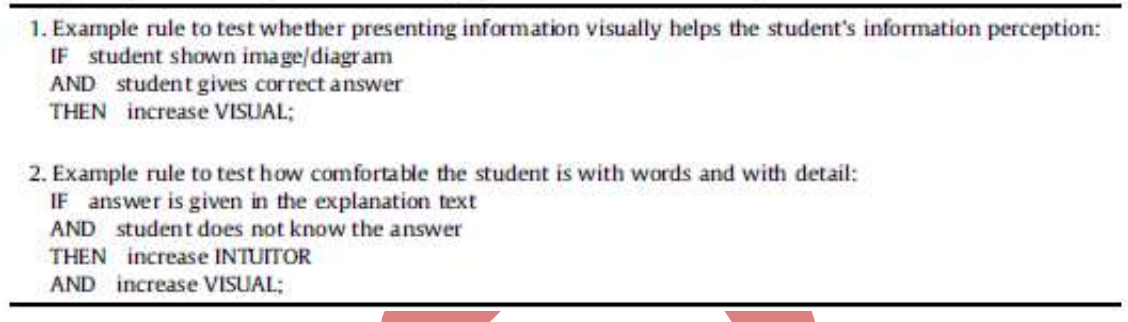

Fig.2 Example Rules to adjust student learning style

\subsection{Immediate Meta Cognitive Feedback will help students acquire better help seeking skills} Immediate meta-cognitive feedback will help students acquire better help seeking skills [4]. Knowing when and how to seek help during learning is a key self-regulatory skill. Research in classrooms suggests that adaptive help-seeking behavior helps students learn more effectively. Effective help seeking has also been shown to be associated with better learning in educational technologies. Here the focus is on help seeking within an intelligent tutoring system, that is, a system that provides step by-step level guidance as students practice a complex problem solving skill.

\begin{tabular}{llll}
\hline Hint & Hint text & Type of hint \\
\hline level & "Enter the value of the radius of circle A" & $\begin{array}{l}\text { Orientation } \\
\text { Instrumental help }\end{array}$ \\
& $\begin{array}{l}\text { "How can you calculate the value of the } \\
\text { radius of circle A given the value of the } \\
\text { diameter of circle A?" } \\
\text { "The radius of a circle is half } \\
\text { of the diameter" }\end{array}$ & Instrumental help \\
4. & "The radius of circle A = 46.5" & Bottom-out hint \\
\hline
\end{tabular}

Fig.3 Hint level in Geometry Cognitive Tutor

Students often can ask for multiple levels of hints for each step. Here an example hints level in the geometry cognitive tutor.

\subsection{Question-Driven competition-based approach}

A detailed description about competition based learning [3] were discussed, which is an ITS that enables competition among students in an educational environment. The paper shows the different features of the tool that they developed for competition based leaning and how they are innovative with respect to previous educational competitive systems. The tool allows the competition among students for improving their learning process in a course. This takes some ideas from the Swiss-system widely used in chess and adapts them to the educational area for competition based approach in learning. The competition is based on different tournaments and rounds. In each round, students are assigned in pairs of two, which compete one against another, and each pair receives different questions that students have to solve in a limit of time.

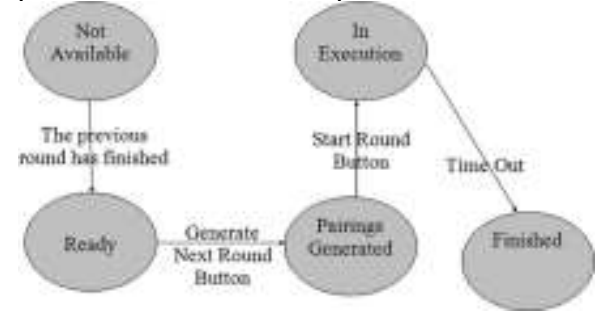

Fig.4 Sequence Flow of competition

The sequence flow of competition is given in fig.4. Students can see their partial ratings after each round and their final rating after a tournament. The tool includes problem solving capabilities with a specific scoring calculation, artificial intelligence capabilities (for the adaptation of the different pairings for each round, the adaptation of problems depending on the students' knowledge level, the on-live visualization of matches, or the different ratings), and management capabilities (with the defined life cycles for tournament or rounds). 


\subsection{Usage of software agent for Politeness Feedback}

Different type of software agents are used to provide politeness interaction in the intelligent tutoring system. Scooter the tutor [1] uses a politeness software agent which dynamically interacts with the students. The response of the students to the animated software agent varies with human tutor. When tutor insults the students, the reaction of the students may change and it can affect the student's learning environment.
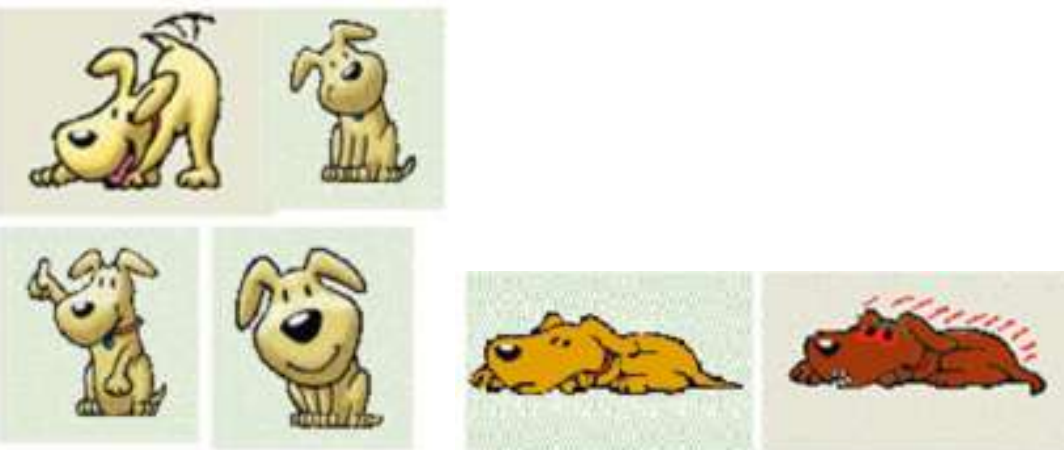

Fig.5 Happy (First), Sad (Second) and Angry (Third) expressions of scooter

But the software agent frequently predicts the student cognition and provides a polite interaction to the students. Here an animated dog is used to interact with the students. The dog shows happy expressions when the students perform well and it shows sad expression when students don't perform well. The proper message is send to the students without affecting the learning environment of the students. Hence different types of animations are shown for different type of message. Fig 5 illustrate the expressions of the tutor agent is given

\subsection{Issues from Prior Research}

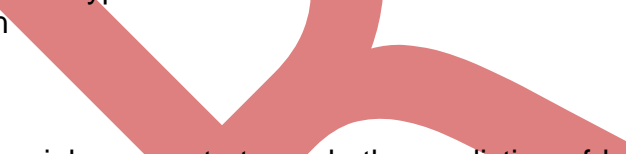

- The automatic prediction of learning style mainly concentrates only the prediction of learning style in intelligent tutoring system. It does not deal with the usage of the predicted style.

- The usage of politeness based agents involves an animated character which expresses different type of expression. But the message that trying to convey to the student doesn't easily reach the student.

- The competition based learning provides deeper competitions which enable a learner to be a part of the game always and He must be alive until the tournament is over. It will take long time to complete the competition since the user compete with all the students who are all participating competition.

- All the above system, each provides a specialized service such as learning styles, competition based learning, providing polite feedback and game based learning [6]. It works separately to increase the learning skill of the students. Hence an power pack system can be developed which concentrate on learning style involves content adaption, polite feedback, Game based learning and competition based learning, an animated polite based agent and a simple quiz with which tries its maximum to increase the cognitive skill of the learners and well known about a subjective knowledge.

\section{RESEARCH PROPOSAL}

\subsection{Introduction}

This paper has presented an adaptive and interactive agent based intelligent tutoring system tool ITSCARE. It imitates the classroom teaching by integrating the intelligent tutoring techniques. It helps to improve the cognitive ability of the student with motivation. The tool focuses on the subject Data structure that places a vital role in computer science domain. ITSCARE provides different types of course materials which are dynamically adapted. It also increases the confidence level of the learners and provides an effective learning experience. The work has been done in analyzing different behaviors for predicting learning styles and cognitive ability. Student's cognitive skill is assessed through Fuzzy algorithm.

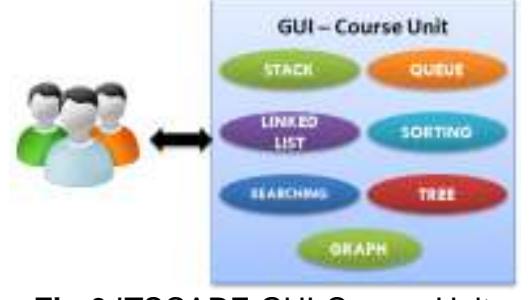

Fig.6 ITSCARE GUI-Course Unit

ITSCARE provides different types of course material in data structure subject such as theoretical based, example based, practical based and Gaming based in a distributed system. ITSCARE GUI-Course unit consist of data structure concepts such as sorting, searching, linked list, stack, queue, tree, graph, and etc. At first, the learner can choose any type of course content for a chapter. At the end of the chapter, the assessment test will be conducted to predict the cognitive ability of the student and learning style. Based on the cognitive prediction, we can improve the cognitive by giving the polite feedback and competition based approach and game based motivation. Game based learning in data structure is 
used to improve motivation among the students and to bring the interest on the subject. The concept in the chapters are converted into games and made to solve by the learner which gives a clear view about the concept of a chapter.

\subsection{ITSCARE Architecture}

The architecture of ITSCARE is illustrated in fig 6. ITSCARE comprises of four agent such as Tutor agent, Test agent, Monitor agent and Game agent. Each agent will perform their specified tasks which are discussed below. Agents communicate with each other through ACL.
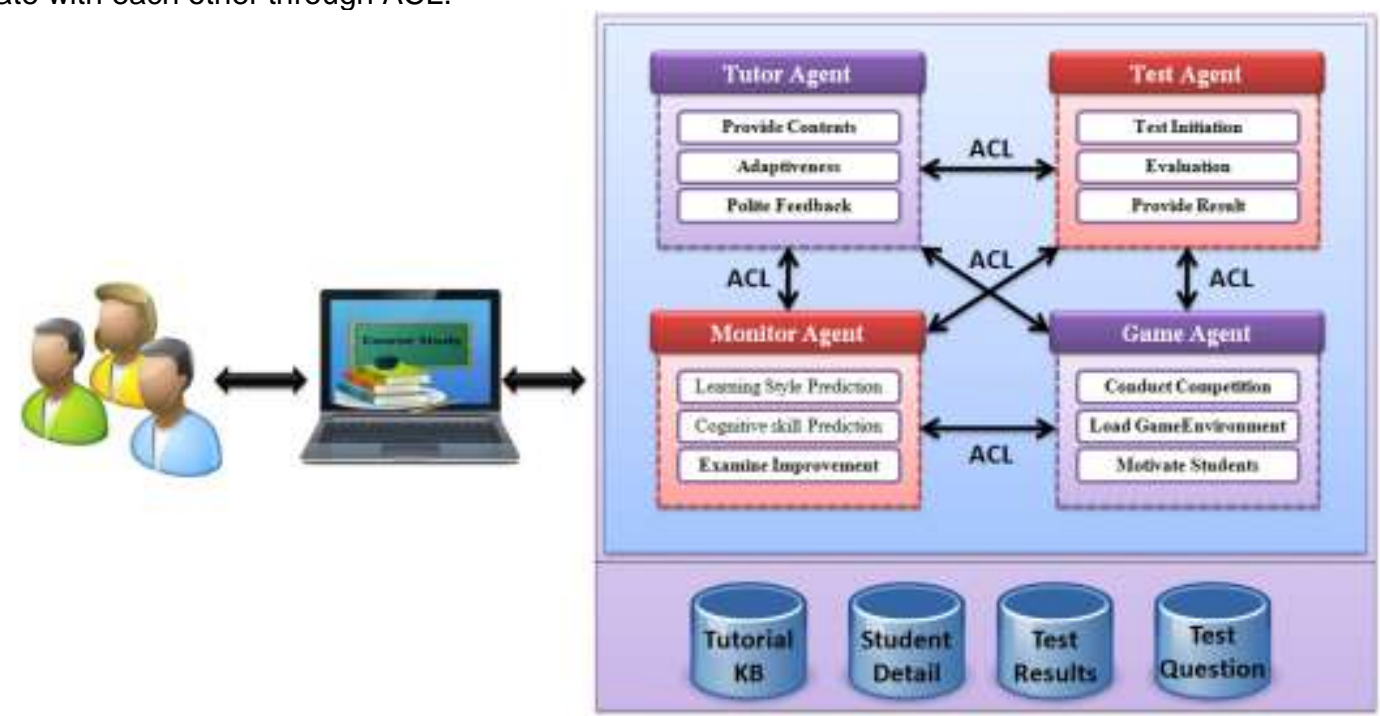

\subsection{Roles of agent}

\section{Tutor agent:}

- Interacts with the students

- Provide content as per the instruction given by monitor agent

- Provide polite feedback

Test agent:

- Conducts the test chapter wise

- Evaluate the answer

- Sends the results to monitor agent and tutor agent

\section{Monitor agent:}

- Tells the tutor agent that what kind of content should be provided to the student using test values

- Cognitive skill of the student is predicted using test values

- Does the complete assessment of students

\section{Game agent:}

- Conducts competition

- Provide games to play by students

\subsection{Polite based feedback}

Tutor agent is responsible for the polite based feedback. Each and every interaction of student to ITSCARE is done by tutor agent. Providing content, cognition result and test result. Hence all the results and feedbacks are provided to the students in a polite manner. An animated cartoon character with audio based feedback is given to the feedback which may not affect the students by its feedback. It congrats the students with a smiley face and brisk voice, when good mark is scored and shows a sad face with dull voice when the students perform low.

\subsection{Conduction of test}

At the completion of each chapter an test is conducted by Test Agent. Test consists of 10 questions. These questions are framed in such a manner for assessing the cognitive skill. Based on that Tutor Agent adapting the content. Cognitive of the learners can be assessed through four factors memory, problem solving, understanding and planning.

These 10 questions are distributed such that 4 questions come under memory, 3 questions come under problem solving and 3 questions come under understanding and planning. For every correct answer from student, 10 marks are 
awarded to the students. For every wrong answer, 0 marks will be awarded. Every Question has consisted of 5 hints. The first hint will provide a clue to find the answer and the last hint will show the answer. As per the usage of hint, the mark of the student is reduced.

Table.2 Hint level and Mark reduction for each question in ITSCARE

\begin{tabular}{lc}
\hline Hint Level & Marks Reduction \\
\hline First & 2 \\
Second & 4 \\
Third & 6 \\
Forth & 8 \\
Fifth & 9 \\
\hline
\end{tabular}

The above table represents the reduction of mark according to the hint used. The usage of first hint reduces 2 marks, second hint reduces 4 marks, third hint reduces 6 marks, fourth hint reduces 8 marks and fifth hint reduces 9 marks. A flow chart based on help seeking model is given in figure 7 .

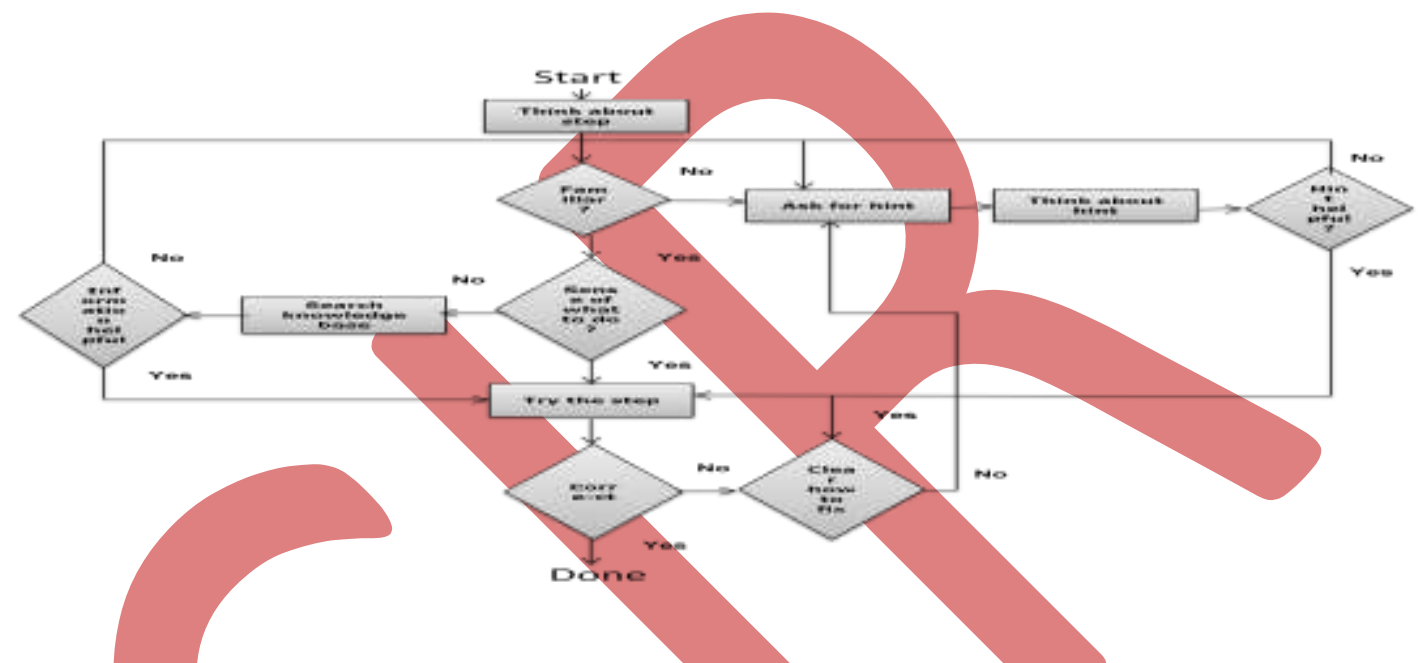

Fig.8 Help seeking based model for Cognitive skill prediction - A Flow Chart

For example, if the student answer a question by using 2 hints. Then the mark awarded to him is 6 . By this way, we will get 10 values of 10 questions totally.

\subsection{Fuzzification of Cognitive skill}

Cognitive skill prediction is done by monitor agent. It gets the parameter from test agent. These 10 values related to the cognitive components are used to predict the cognitive skill of the student such that low, medium and high. The values may range from 0 to 10 and it is difficult to predict the cognitive skill of the students hence we use fuzzy logic.

Fuzzy logic is a form of many-valued logic. The fuzzy logic variables may have a truth value that ranges in degree between 0 and 1. Fuzzy logic has been extended to handle the concept of partial truth, where the truth value may range between completely true and completely false. Fuzzy logic is given with 3 parameter values such as marks of memory, problem solving, understanding and planning.

Table.3 Score and its level

\begin{tabular}{cc}
\hline Score Value & Level \\
\hline $0-3$ & Low \\
$4-7$ & Medium \\
$8-10$ & High \\
\hline
\end{tabular}

The above table represents the set representation of value. 0 to 3 represent low, 4 to 7 represent medium and 8 to 10 represent high.

Hence the fuzzy logic is represented by

If(skill==0.0 to skill==0.3)

The cognitive skill is low

Else if(skill $==0.4$ to skill $==0.7$ )

The cognitive skill is medium

Else 
The cognitive skill is high //(skill==0.8 to skill==1.0)

The overall cognitive skill value is calculated by predicted fuzzy value explained in table 4.

Table.4 Factors and their result using Fuzzy approach

\begin{tabular}{lllll}
\hline Memory & $\begin{array}{l}\text { Problem } \\
\text { Solving }\end{array}$ & $\begin{array}{l}\text { Understanding And } \\
\text { Planning }\end{array}$ & $\begin{array}{l}\text { Predicted } \\
\text { Fuzzy Value }\end{array}$ & $\begin{array}{l}\text { Overall } \\
\text { Result }\end{array}$ \\
\hline Low & Low & Low & 0.2 & Low \\
Medium & Medium & Medium & 0.6 & Medium \\
High & High & High & 0.99 & High \\
Low & Low & Medium & 0.2 & Low \\
Low & Low & High & 0.6 & Medium \\
Medium & Medium & High & 0.6 & Medium \\
Medium & Medium & Low & 0.6 & Medium \\
High & High & Low & 0.6 & Medium \\
High & High & Medium & 0.9 & High \\
\hline
\end{tabular}

The sample marks obtained by a 3 students are given below based on the factors specified in the table 5 .

Table.5 Fuzzification of Test Score - An Example

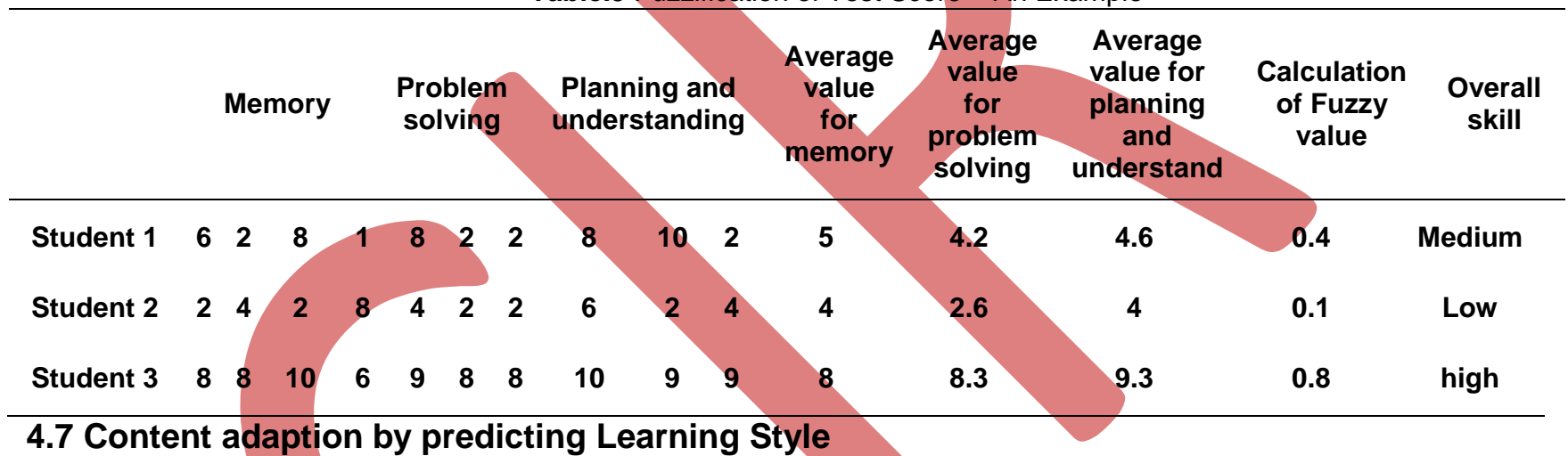

The monitor agent is responsible for the content adaption. The parameters for content adaption are test marks which is provided by the test agent. The monitor agents calculate and provide the result to the tutor agent such that what type of content should be provided to the student.

The following calculation is made by monitor agent:

$$
\frac{\text { Correct Practical Questions }}{\text { Total Practical Questions }} \text { compared to } \frac{\text { Correct Theoretical Questions }}{\text { Total Theoretical Questions }}
$$

Typical learner behavior characteristics extracted from the ILS model. Where participants performed better in practical questions, the system predicts the learning style to be Active and Sensory. Participants who performed better in theoretical questions were predicted to be Reflective and Intuitive. This is used to predict the perception (Sensory/Intuitive) and processing (Active/Reflective) ILS dimensions. Then learners will get the theoretical based content if the correct theoretical questions value is high because Sensory and Intuitive learners will always prefer theoretical contents. Else the learners will get the practical based content if the correct practical questions value is high since Active/Reflective learner prefers practical and real data content.

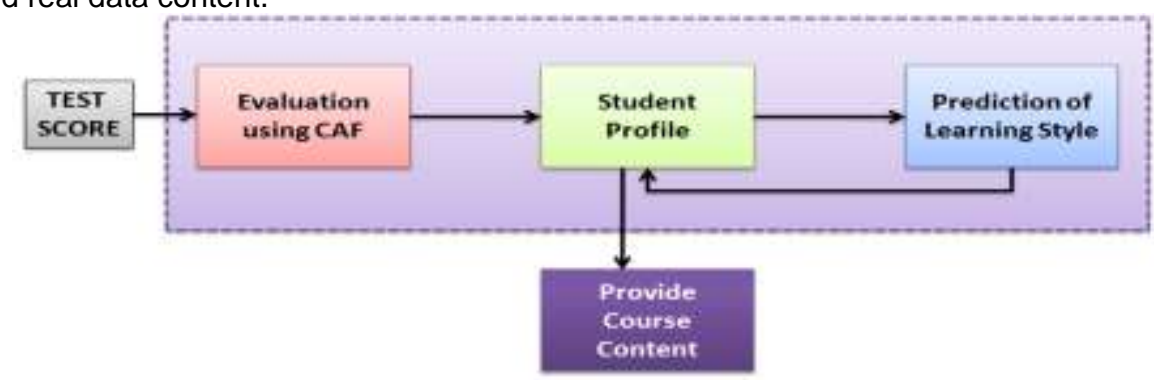

\subsection{Motivation through Games}

Fig.9 Content Adaption

Game agent is responsible for the game based learning. Motivation is an important factor that directly reflects on cognition. The result of the cognition is based on the interest factor of the students towards the subject. Each content has 
a unique timeslot to read the topic. When a student cross the unique timeslot. Then it is concluded that the student is not interested in the concept and he needs motivation. A game environment is created and allows the student to play the game. For example, the student is currently studying the topic named "sorting".

\begin{tabular}{ll}
\multicolumn{2}{l}{ Table. 6 Game and its Timing to start } \\
\hline Sorting & Timeslot \\
\hline Theory based & 20 mins \\
Example based & 10 mins \\
Video based & 10 mins \\
\hline
\end{tabular}

The above table represents the unique timestamp of a topic which may differ by the content type. It express that theory based content can be viewed only 20 mins, example based content can be viewed only 10 mins and video based content can be viewed only 10 mins.

The student crosses 20 min for theory based content, and game related to previous concept is recommended such as "Searching". Hence motivation directly reflects on interest, the interest of the student towards the topic is created.

\subsection{Cognition Improvement}

Competition is one of the most important of ITSCARE. On every day, on a particular time, a competition will be initiated and any one student who is online can participate. A common set of questions will be given to the participants and the competition result is saved and continuously monitored. Hence by participating in a competition a student can improve his cognitive skill.

\section{CONCLUSION AND FUTURE WORK}

This work discuss about the detailed description of ITSCARE. It helps the student to learn the subjects easily without the intervention of human teacher. It enables students to apply their creativeness and experience a effective learning environment. We use JADE platform to implement agents. ITSCARE tutors Data Structure. This work can be enhanced by deploying in distributed environment and also predicting all the styles of learning model.

\section{References}

[1] Ma. Mercedes T. Rodrigo, Ryan S.J.D. Baker, Jenilyn Agapito, Julieta Nabos, Ma. Concepcion Repalam, Salvador S. Reyes Jr., "The Effects Of An Interactive Software Agenton Student Affective Dynamics While Using An Intelligent Tutoring System", IEEE Transactions On Affective Computing,2012

[2] Annabel Latham , Keeley Crockett , David Mclean , Bruce Edmonds ,"A Conversational Intelligent Tutoring System To Automatically Predict Learning Styles",ELSEVIER,2012

[3] Pedro J. Muñoz-Merino, Manuel Fernández Molina, Mario Muñoz-Organero, Carlos Delgado Kloos,"An Adaptive And Innovative Question-Driven Competition-Based Intelligent Tutoring System For Learning",ELSEVIER,2012

[4] Ido Roll, Vincent Aleven, Bruce M. Mclaren, Kenneth R.Koedinger 'Improving Students' Help- Seeking Skills Using Metacognitive Feedback In An Intelligent Tutoring System"Elsevier,2011.

[5] Cristina Conati “Intelligent Tutoring System: New Challenges And Directions", 2010

[6] Lotfi Derbali, Pierre Chalfoun \& Claude Frasson, "A Theoretical And Empirical Approach In Assessing Motivational Factors : From Serious Game TO An ITS,2011

[7] Peter Brusilovsky, "Adaptive and Intelligent Web-based Educational Systems" , International Journal of Artificial Intelligence in Education 13, 2003

[8] Ioannis Magnisalis, Stavros Demetriadis, And Anastasios Karakostas, "Adaptive And Intelligent Systems For Collaborative Learning Support: A Review of The Field",IEEE TRANSACTIONS ON LEARNING TECHNOLOGIES,2011

[9] Chih-Yueh Chou, Bau-Hung Huang, Chi-Jen Lin ,"Complementary Machine Intelligence And Human Intelligence In Virtual Teaching Assistant For Tutoring Program Tracing",Elsevier,2011.

[10] Ido Roll, Vincent Aleven, Bruce M. Mclaren, Kenneth R.Koedinger"Improving Students' Help-Seeking Skills Using Metacognitive Feedback In An Intelligent Tutoring System"Elsevier,2011.

[11] Bruce M.Mclaren, Krista E.Deleeuw, Richard E. Mayer " A Politeness Effect In Learning With Web-Based Intelligent Tutors" Elsevier,2011.

[12] Samy S. Abu Naser,"Predicting Learners Performance Using Artificial Neural Networks In Linear Programming Intelligent Tutoring System",International Journal Of Artificial Intelligence \& Applications (IJAIA),2012

[13] Vladoglavinic, Marko Rosie, Marijazelic "Extending Intelligent Tutoring Systems To Mobile Devices" Springer, 2008.

[14] S. Abu-Naser, A. Ahmed, N. Al-Masri "An Intelligent Tutoring System For Learning Java Objects" International Journal Of Artificial Intelligence \& Applications,2011.

[15] Moe Moe Aye And Mie Mie Thet Thwin “Mobile Agent Based Online Examination System”,IEEE 2008.

[16] T.T. Gohkinshuk “A Discussion On Mobile Agents Based Mobile Web-Based ITS” ,IEEE2002. Author' biography 


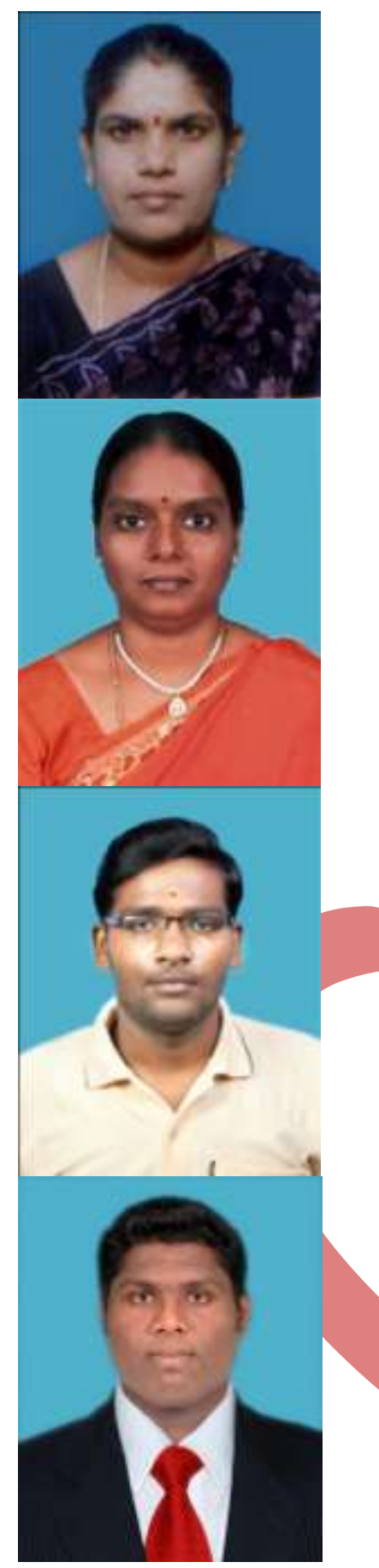

R. Gowri received her B.E from Madras University, M.Tech. Degree in Computer Science \& Engineering from Pondicherry University, and pursuing Ph.D (Computer Science \& Engineering) in Pondicherry Engineering College, Pondicherry University, Puducherry, India. She is currently working as Associate Professor, Department of Information Technology, Sri Manakula Vinayagar Engineering college (Pondicherry University), Puducherry. Her research interests are in the areas of Database Management Systems, Algorithms, and Agent Technology. She has published 25 papers in reputed journal and conferences. She is a member of computer society of India, ISTE

S. Kanmani received her B.E and M.E in Computer Science and Engineering from Bharathiar University and PhD from Anna University, Chennai. She has been the faculty of department of Computer Science and Engineering, Pondicherry Engineering College from 1992. Presently she is heading the department of in Information Technology. Her research interests are in software engineering, software testing and object oriented systems. She is a member of computer society of India, ISTE and institute of engineers India. She has published about 120 papers in international conferences and journals.

M. Santhosh pursuing his B.Tech (Information Technology) in Sri Manakula Vinayagar Engineering College affiliated to Pondicherry University, Puducherry, India. His research interest are in Computer Networks, Database Management Systems and Object Oriented Programming.

S. Naresh pursuing his B.Tech (Information Technology) in Sri Manakula Vinayagar Engineering College affiliated to Pondicherry University, Puducherry, India. His research interest are in Database Management Systems, Software Engineering and Object Oriented Programming. 